Published in final edited form as:

Science. 2018 November 30; 362(6418): 1055-1060. doi:10.1126/science.aau6509.

\title{
Human tumor genomics and zebrafish modeling identify SPRED1 loss as a driver of mucosal melanoma
}

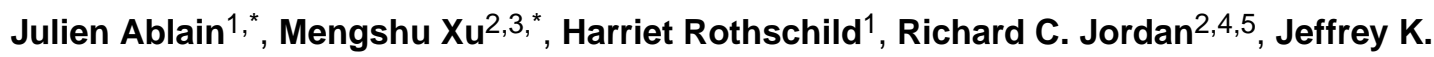 \\ Mito $^{1,6}$, Brianne H. Daniels ${ }^{2,4}$, Caitlin F. Bell ${ }^{7}$, Nancy M. Joseph ${ }^{4}$, Hong Wu ${ }^{8}$, Boris C. \\ Bastian $^{2,3, \dagger}$, Leonard I. Zon ${ }^{1,9,10, \dagger, \ddagger}$, and Iwei Yeh ${ }^{2,3, \dagger, \ddagger}$ \\ 1Stem Cell Program and Division of Hematology/Oncology, Boston Children's Hospital and Dana- \\ Farber Cancer Institute, Boston, MA 02115, USA. \\ ${ }^{2}$ Helen Diller Family Comprehensive Cancer Center, University of California, San Francisco,San \\ Francisco, CA 94158, USA. \\ ${ }^{3}$ Department of Dermatology, University of California, San Francisco, San Francisco, CA 94143, \\ USA. \\ ${ }^{4}$ Department of Pathology, University of California, San Francisco, San Francisco, CA 94143, \\ USA. \\ ${ }^{5}$ Department of Orofacial Sciences, University of California, San Francisco, San Francisco, CA \\ 94143, USA. \\ ${ }^{6}$ Department of Pathology, Brigham and Women's Hospital, Boston, MA 02115, USA. \\ ${ }^{7}$ Department of Medicine, Stanford University Medical Center, Stanford, CA 94305, USA. \\ ${ }^{8}$ Fox Chase Cancer Center, Philadelphia, PA 19111, USA. \\ ${ }^{9}$ Harvard Stem Cell Institute, Harvard University, Cambridge, MA 02138, USA. \\ ${ }^{10}$ Howard Hughes Medical Institute, Boston Children's Hospital and Harvard University, Boston, \\ MA 02115, USA.
}

\footnotetext{
${ }^{\ddagger}$ Corresponding author. iwei.yeh@ucsf.edu (I.Y.); leonard. zon@enders.tch.harvard.edu (L.I.Z.). Author contributions: I.Y., J.A., M.X., B.C.B., and L.I.Z. designed the study. I.Y., B.C.B., H.W., N.M.J., B.H.D., and R.C.J. performed sample selection and collection. M.X. and I.Y. performed genetic analyses. M.X. and I.Y. performed immunohistochemistry, and I.Y. analyzed the results. J.A., H.R., and C.F.B. performed zebrafish experiments, and J.A. and J.K.M. analyzed the results. J.A. performed human melanoma cell line experiments and analyzed the results. I.Y., J.A., M.X., B.C.B., and L.I.Z. wrote the manuscript, and all authors provided constructive feedback.

*These authors contributed equally to this work.

${ }^{\dagger}$ These authors contributed equally to this work.

Competing interests:I.Y. received grants from Ignyta, Novartis, and Bayer, outside the submitted work. B.C.B. is a consultant for Lilly, Inc., outside the submitted work. L.I.Z. is a founder and stockholder of Fate Therapeutics, CAMP4 Therapeutics, and Scholar Rock, outside the submitted work. All other authors declare no conflicts of interest.

Data and materials availability: Mucosal melanoma targeted DNA sequencing data have been deposited in dbGAP (project phs001594.v1). All plasmids described in this study have been deposited at Addgene.

SUPPLEMENTARY MATERIALS

www.sciencemag.org/content/362/6418/1055/supp1/DC1 Materials and Methods

Figs. S1 to S12

Tables S1 to S4

References (30-70)
} 


\section{Abstract}

Melanomas originating from mucosal surfaces have low mutation burden, genomic instability, and poor prognosis. To identify potential driver genes, we sequenced hundreds of cancer-related genes in 43 human mucosal melanomas, cataloging point mutations, amplifications, and deletions. The SPRED1 gene, which encodes a negative regulator of mitogen-activated protein kinase (MAPK) signaling, was inactivated in $37 \%$ of the tumors. Four distinct genotypes were associated with SPRED1 loss. Using a rapid, tissue-specific CRISPR technique to model these genotypes in zebrafish, we found that SPRED1 functions as a tumor suppressor, particularly in the context of KIT mutations. SPRED1 knockdown caused MAPK activation, increased cell proliferation, and conferred resistance to drugs inhibiting KIT tyrosine kinase activity. These findings provide a rationale for MAPK inhibition in SPRED1-deficient melanomas and introduce a zebrafish modeling approach that can be used more generally to dissect genetic interactions in cancer.

Identification of driver genes in cancer is necessary for the development of precision therapies. Whereas sequencing of human tumors can point to potential oncogenes or tumor suppressors, robust animal models aid in implicating candidate drivers in malignant transformation. Discovery of new cancer genes and treatments is often hindered by the speed at which a model can be generated. In melanoma, certain genetic subtypes, including "triple wild-type" tumors (i.e., wild-type for BRAF, NRAS, and NF1) lack reliable models altogether. Developing new animal models through regular transgenesis and breeding is time consuming and labor intensive. There is a need for new and efficient modeling approaches to study cancer genes in vivo.

One tumor type in which genetics has not been fully explored is mucosal melanoma, which originates from melanocytes within mucosal epithelia (1-3). Mucosal melanomas harbor few point mutations, in keeping with their primary location on sun-protected sites $(4,5)$. Instead, mucosal melanomas display genomic instability characterized by amplifications and deletions $(6,7)$. Although dwarfed by the incidence of ultraviolet-induced melanomas in white populations, mucosal melanoma exhibits uniform incidence across ethnic groups and accounts for 9 to $22 \%$ of melanomas in Asian and black populations (8-10). Mucosal melanoma has a poor prognosis, with a 5-year survival rate of around 33\% (11). In contrast to cutaneous melanomas, most mucosal melanomas do not harbor a known mitogenactivated protein kinase (MAPK) activating alteration. Mucosal melanomas harbor activating KIT mutations in $15 \%$ of cases, inactivation of NF1 in 13\% of cases, and BRAF [V600E $\left.\left(\mathrm{Val}{ }^{600} \rightarrow \mathrm{Glu}\right)\right]$ or NRAS $\left[\mathrm{G} 12\left(\mathrm{Gly}^{12}\right), \mathrm{G} 13\left(\mathrm{Gly}^{13}\right)\right.$, or Q61 $\left.\left(\mathrm{Gln}^{61}\right)\right]$ mutations in 6 and $8 \%$ of cases, respectively $(7,12)$ (see table $\mathrm{S} 1$ for a summary of mucosal melanoma molecular studies). Treatment of KIT-mutant melanomas with inhibitors of KIT tyrosine kinase activity produces short-lived clinical responses (13-15). Thus, few patients with mucosal melanoma currently benefit from targeted therapies.

To better understand mucosal melanomas and to identify additional oncogenic alterations and therapeutic strategies, we extracted DNA from formalin-fixed paraffin-embedded primary or metastatic mucosal melanomas from 43 patients and performed targeted sequencing to high coverage (median of 300-fold mean coverage; see table S2 for clinical features and table $\mathrm{S} 3$ for sequencing metrics) (16). The average somatic mutation burden 
(calculated from 10 cases for which normal, nonlesional tissue was also sequenced) was low compared to other cancers (1.5 mutations/Mb), consistent with prior studies. Genomic instability in mucosal melanomas, as measured by two established metrics, was one of the highest among cancers (fig. S1). For each case, we identified point mutations, amplifications, and deletions known to be pathogenic in cancer (Fig. 1A). Alterations in BRAF (16\%), RAS isoforms (16\%), or NF1 (14\%), which account for MAPK-activating mutations in $86 \%$ of cutaneous melanomas (17), were collectively found in only $46 \%$ of mucosal melanomas. BRAF mutations consisted mainly of noncanonical mutations [L505H, G469A, L597R, and T599I (L, Leu; H, His; A, Ala; R, Arg; T, Thr; I, Ile)] (18-20) known to lead to weaker MAPK pathway activation than BRAF V600E. Activating KIT mutations occurred in $12 \%$ of cases. The distribution of mutations activating the MAPK pathway thus differs dramatically from that seen in cutaneous melanoma (Fig. 1B).

We identified genomic regions that are recurrently amplified and deleted across samples by GISTIC2 analysis, revealing a frequently deleted region at chromosome band 15q14, occurring in 21\% of cases (Fig. 1C). All deletions of 15q14 included the SPRED1 locus, and in three cases, SPRED1 was the only gene within the deleted region. SPRED1 (sproutyrelated, EVH1 domain-containing protein 1) is a negative regulator of the RAS-MAPK pathway $(21,22)$. We estimated copy number at the SPRED1 locus and classified SPRED1 deletion as deep (nine cases) or shallow (four cases) (Fig. 2A and fig. S2). In two cases with shallow deletions of SPRED1, the remaining SPRED1 alleles harbored truncating mutations, implicating SPRED1 as the tumor suppressor gene at 15q14. SPRED1 biallelic inactivation by deep deletion or by truncating mutation combined with loss of the wild-type allele affected $26 \%$ of cases (11/43), a frequency that is significantly greater than that in cutaneous melanoma $\left(2.4 \%\right.$, Fisher's exact test, $\left.\mathrm{P}=8.7 \times 10^{-7}\right)$.

To confirm loss of SPRED1 protein expression in tumors with SPRED1 biallelic inactivation, we performed immunohistochemistry for SPRED1 on 26 cases ( 8 with biallelic loss, 2 with monoallelic loss, and 16 without evidence of genetic inactivation of SPRED1) for which leftover tissue was available. In a melanocytic nevus that we used as the positive control, SPRED1 immunoreactivity was mainly in the cytoplasm, with accentuation near the membrane, in keeping with previous reports of SPRED1's subcellular localization (21). In all eight cases with inactivation of both SPRED1 alleles, the majority of tumor cells did not show any immunoreactivity. Of the 16 mucosal melanomas in which we did not detect alterations of the SPRED1 gene, eight displayed SPRED1 immunoreactivity similar to the positive control, whereas four had cytoplasmic staining and four had no SPRED1 immunoreactivity (Fig. 2B, fig. S3, and table S4). The absence of SPRED1 immunoreactivity in cases without SPRED1 biallelic inactivation suggests that SPRED1 was inactivated in these tumors by mechanisms not detected by our sequencing platform, such as by structural rearrangements within noncoding regions of SPRED1 or by epigenetic silencing. Overall, $37 \%$ of the 43 mucosal melanomas we examined showed evidence of SPRED1 loss of function (biallelic inactivation and/or absence of SPRED1 protein by immunohistochemistry).

SPRED1 recruits NF1 to the plasma membrane, where NF1 catalyzes the conversion of active RAS-GTP (guanosine triphosphate) to the inactive RAS-GDP (guanosine 
diphosphate) state (23-25). Our analysis shows that NF1 biallelic loss-of-function mutations (12\%) occurred in a mutually exclusive pattern with SPRED1 loss. Although this pattern of mutual exclusivity did not reach statistical significance, the functional relationship between SPRED1 and NF1 suggests that SPRED1 inactivation and NF1 inactivation play similar roles as driver mutations in mucosal melanoma. Altogether, complete inactivation of either NF1 or SPRED1 occurred in at least $49 \%$ of our cohort of tumors.

We next analyzed the genetic context of SPRED1 inactivation in melanoma. Most of the cases with SPRED1 loss (either by biallelic activation or loss of protein expression) were triple wild type (i.e., without activating mutations in BRAF, RAS isoforms, or biallelic loss of NF1), both in our cohort of mucosal melanomas and in The Cancer Genome Atlas (TCGA) cohort of cutaneous melanomas. Notably, 30\% of cases with SPRED1 loss (7/23) also harbored KIT alterations (Fisher's exact test, $\mathrm{P}=7.8 \times 10^{-6}$ ) (Fig. 2C). Another five cases with SPRED1 loss harbored alterations in BRAF or NRAS, and three cases had PTEN deep deletions. This pattern suggests that SPRED1 inactivation may cooperate with other oncogenic events that activate the MAPK or phosphoinositide 3-kinase (PI3K) pathway.

To assess the function of SPRED1 in melanoma in vivo, we created MAZERATI (Modeling Approach in Zebrafish for Rapid Tumor Initiation), a platform to rapidly model potential cancer drivers in vivo. Leveraging the power of trans-genesis in zebrafish, we developed a robust method to express oncogenes and inactivate candidate tumor suppressor genes specifically in the melanocytes of the zebrafish using the MiniCoopR vector (26). We adapted our tissue-specific CRISPR system (27) to create the CRISPR MiniCoopR vector; this vector expresses Cas 9 under the control of the melanocyte-specific mitfa promoter and contains a mitfa minigene that rescues melanocyte formation in a mosaic fashion upon injection into mitfa ${ }^{-/}$embryos (Fig. 3A). We generated MiniCoopR vectors expressing the oncogenes KIT K642E (K, Lys), BRAF V600E, or NRAS Q61R, and CRISPR MiniCoopR vectors targeting the tumor suppressors tp53, pten, or cdkn2a. Except for the vector expressing NRAS Q61R, none of these vectors alone initiated cancer in adult casper zebrafish (28), pointing to the need for both oncogene expression and tumor suppressor loss to trigger melanoma formation (Fig. 3, B to E). Accordingly, combining a MiniCoopR vector expressing KIT K642E, BRAF V600E, or NRAS Q61R and a CRISPR MiniCoopR vector targeting tp53 rapidly yielded melanomas (Fig. 3, B to E, and fig. S4). Of note, the anatomic location of KIT-mutant melanomas differed significantly from that of BRAF and NRAS mutant melanomas, as KIT-mutant melanomas occurred on the fins and internally more often than BRAF and NRAS mutant melanomas (fig. S4H). Inactivation of cdkn2a also cooperated with each of the three oncogenes to generate melanomas in vivo, whereas pten loss initiated melanoma formation in combination with BRAF V600E but not KIT mutants (figs. S5 and S6), pointing to differential impacts of PI3K pathway activation depending on the driving oncogene. These results indicate that our dual-vector MAZERATI system enables efficient and rapid generation of genetically defined primary tumors in $\mathrm{F}_{0}$ adult animals within 3 months and can be used to test the effects of multiple combinations of onco-genes and tumor suppressor genes on tumor-igenesis. In particular, we were able to model multiple genetic contexts in which SPRED1 was lost in melanoma. 
To determine the effects of SPRED1 loss in these different contexts, we inactivated spred1 using the CRISPR MiniCoopR vector in various zebrafish melanoma models. Targeting of spred1 in combination with tp53 did not result in melanoma formation within a year postinjection (Fig. 3E), suggesting that SPRED1 loss is a weaker driver than KIT, BRAF, or NRAS mutation. Targeting spred1 in combination with either KIT K642E or BRAF V600E also failed to initiate melanoma, indicating that SPRED1 loss cannot substitute for the inactivation of tumor suppressor genes like TP53 or CDKN2A (Fig. 3, B and C). Targeting spred1 in the KIT L576P/tp53 (P, Pro) or KIT K642E/tp53 contexts significantly accelerated the onset of melanoma in our zebrafish model (Fig. 3B and fig. S7). Eleven of 14 (79\%) KIT $\mathrm{K} 642 \mathrm{E} / \mathrm{tp} 53 /$ spred1 tumors tested displayed clonal biallelic inactivation of spred1 as assessed by sequencing of the CRISPR target sites. By contrast, spred1 inactivation in the BRAF V600E/tp53 or NRAS Q61R/tp53 contexts did not significantly affect melanoma onset (Fig. 3, C and D). Spred1 inactivation in zebrafish melanocytes also accelerated tumor onset in the KIT K642E/cdkn2a context (fig. S7). Conversely, SPRED1 overexpression using the MiniCoopR vector significantly delayed melanoma development in vivo in the context of mutant KIT but not in the context of mutant BRAF (fig. S8). Targeting spred1 in addition to pten and tp53 resulted in late-onset melanomas, which were not observed when targeting pten and tp53 alone (Fig. 3E). Thus, spred1 inactivation accelerated melanoma onset in zebrafish in combination with mutant KIT expression or pten inactivation, but not mutant BRAF or NRAS expression, in the setting of tp53 inactivation. These results establish SPRED1 as a bona fide tumor suppressor gene in melanoma and demonstrate in vivo cooperation between SPRED1 deletions and activating KIT mutations.

The human melanoma cell line WM3211 harbors a KIT L576P mutation and has intact SPRED1. We found that short hairpin RNA (shRNA)- mediated knockdown of SPRED1 in WM3211 cells increased their proliferation and MAPK activity (Fig. 4, A and B). These effects were not observed upon SPRED1 knockdown in BRAF-driven melanoma cells (fig. S9). Conversely, overexpression of SPRED1 in WM3211 cells decreased their proliferation and MAPK activity (fig. S10). These data indicate that SPRED1 modulates MAPK activation and cell proliferation in KIT-mutant melanoma. SPRED1 down-regulation by shRNA was associated with the persistence of a low, but detectable, level of extracellular signal-regulated kinase (ERK) phosphorylation in WM3211 cells treated with the KIT inhibitor dasatinib (Fig. 4B). SPRED1 knockdown also dampened the anti-proliferative effect of dasatinib (Fig. 4C). We confirmed that this resistance to KIT inhibition was due, at least in part, to enhanced MAPK activity because it could be abolished by trametinibmediated MAPK kinase (MEK) inhibition (Fig. 4D). We validated these results using a CRISPR approach. We found that in cultures of WM3211 cells suboptimally transfected with CRISPR vectors targeting SPRED1, the proportion of SPRED1 frameshift mutant alleles sharply increased over time in dasatinib-treated cultures (Fig. 4E), whereas it modestly increased in vehicle-treated cultures. This result indicates that SPRED1 knock out cells were under strong positive selection in the presence of KIT inhibitor. Finally, to test the effect of SPRED1 loss on treatment of KIT-mutant tumors in vivo, we devised a protocol to treat adult zebrafish daily for 14 days. Dasatinib treatment of zebrafish with KIT-driven melanomas resulted in significant reductions in tumor size (fig. S11). However, the response to KIT inhibition was reduced with spred1 knockout (Fig. 4F and fig. S12). These data 
suggest that SPRED1 loss confers resistance to KIT inhibition in KIT-mutant melanoma by sustaining MAPK signaling and cell proliferation.

The increased incidence of SPRED1 loss and KIT mutation in mucosal as compared to cutaneous melanoma (7) suggests that mucosal melanocytes are more susceptible to transformation by these alterations. The different anatomic distribution of melanomas initiated with BRAF, NRAS, or KIT mutations in our zebrafish model supports the concept of differential responses of melanocytes from different anatomic locations to oncogenic alterations. Our findings indicate that triple wild-type melanomas, in many cases, rely on MAPK hyperactivation mediated by SPRED1 loss, often in the context of mutant KIT.

Our study establishes SPRED1 as a major tumor suppressor gene in mucosal melanoma. Our findings have important consequences for the management of KIT-driven melanoma because they suggest that the loss of negative regulators of the MAPK pathway like SPRED1 reduces the sensitivity of these tumors to KIT inhibition. Loss of SPRED1 in KIT-mutant melanomas may, in part, explain the limited therapeutic efficacy of KIT inhibitors in melanoma as compared to their success in other KIT-mutant cancers, such as gastrointestinal stromal tumor. We show that melanomas that harbor KIT mutations and SPRED1 loss depend on sustained MAPK signaling, indicating that combining KIT and MEK inhibitors may provide clinical benefit for patients with KIT-mutant melanomas. Screening for SPRED1 status in melanoma, particularly those with KIT mutations, may prove useful to inform clinical and therapeutic decisions. Finally, our study illustrates the power of combining genomic analyses of human tumors with in vivo modeling to identify actionable driver alterations in cancer.

\section{Supplementary Material}

Refer to Web version on PubMed Central for supplementary material.

\section{ACKNOWLEDGMENTS}

We thank C. Onodera and E. Talevich for helpful advice during data analysis. Many thanks to S. Liu and E. Martin for excellent technical assistance. We also thank S. Avagyan and M. Fazio for critical reading of the manuscript and helpful discussions.

Funding: Funding for this study was provided by the Melanoma Research Foundation, the Dermatology Foundation, the Melanoma Research Alliance, the Ellison Foundation, the Starr Foundation, the V Foundation, the Terry Patters Melanoma Foundation, and the National Cancer Institute (1R35CA220481 to B.C.B., R01CA103846 to L.I.Z., K99CA201465 to J.A., U24CA196067 to R.C.J., and T32HL007627 to J.K.M.). L.I.Z.is a Howard Hughes Medical Institute Investigator.

\section{REFERENCES AND NOTES}

1. Zak FG, Lawson W, Ann. Otol. Rhinol. Laryngol 83, 515-519 (1974). [PubMed: 4137290]

2. Clemmensen OJ, Fenger C, Histopathology 18, 237-241 (1991). [PubMed: 2045074]

3. Barrett AW, Raja AMH, Arch. Oral Biol 42, 77-81 (1997). [PubMed: 9134118]

4. Furney SJ et al., J. Pathol 230, 261-269 (2013). [PubMed: 23620124]

5. Krauthammer M et al., Nat. Genet 44, 1006-1014 (2012). [PubMed: 22842228]

6. Curtin JA et al., N. Engl. J. Med 353, 2135-2147 (2005). [PubMed: 16291983] 
7. Curtin JA, Busam K, Pinkel D, Bastian BC, J. Clin. Oncol 24, 4340-4346 (2006). [PubMed: 16908931]

8. Byrd-Miles K, Toombs EL, Peck GL, J. Drugs Dermatol. 6, 10-16 (2007). [PubMed: 17373156]

9. Chi Z et al., BMC Cancer 11, 85 (2011). [PubMed: 21349197]

10. Altieri L, Wong MK, Peng DH, Cockburn M, J. Am. Acad. Dermatol 76, 250-257 (2017). [PubMed: 27742175]

11. Kirchoff DD et al., Am. Surg 82, 1-5 (2016). [PubMed: 26802836]

12. Hodis E et al., Cell 150, 251-263 (2012). [PubMed: 22817889]

13. Guo J et al., J. Clin. Oncol 29, 2904-2909 (2011). [PubMed: 21690468]

14. Carvajal RD et al., JAMA 305, 2327-2334 (2011). [PubMed: 21642685]

15. Hodi FS et al., J. Clin. Oncol 31, 3182-3190 (2013). [PubMed: 23775962]

16. Materials and methods are available as supplementary materials.

17. Cancer Genome Atlas Network, Cell 161, 1681-1696 (2015). [PubMed: 26091043]

18. Wan PTC et al., Cell 116, 855-867 (2004). [PubMed: 15035987]

19. Dahlman KB et al., Cancer Discov 2, 791-797 (2012). [PubMed: 22798288]

20. Davies H et al., Nature 417, 949-954 (2002). [PubMed: 12068308]

21. Wakioka T et al., Nature 412, 647-651 (2001). [PubMed: 11493923]

22. Nonami A et al., J. Biol. Chem 279, 52543-52551 (2004). [PubMed: 15465815]

23. Stowe IB et al., Genes Dev 26, 1421-1426 (2012). [PubMed: 22751498]

24. Dunzendorfer-Matt T, Mercado EL, Maly K, McCormick F,Scheffzek K, Proc. Natl. Acad. Sci. U.S.A 113, 7497-7502 (2016). [PubMed: 27313208]

25. Hirata Y et al., J. Biol. Chem 291, 3124-3134 (2016). [PubMed: 26635368]

26. Ceol CJ et al., Nature 471, 513-517 (2011). [PubMed: 21430779]

27. Ablain J, Durand EM, Yang S, Zhou Y, Zon LI, Dev. Cell 32, 756-764 (2015). [PubMed: 25752963]

28. White RM et al., Cell Stem Cell 2, 183-189 (2008). [PubMed: 18371439]

29. Huang FW et al., Science 339, 957-959 (2013). [PubMed: 23348506] 
A

$\frac{\frac{2}{m}}{\frac{0}{\alpha}}$

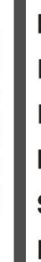

BRAF

NRAS

KRAS

KIT

NF1

SPRED

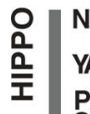

\section{NF2}

YAP1

PAK1/

CDK

CDK

MYC

MDM2

MITF

TERT

SF3B1

Legend

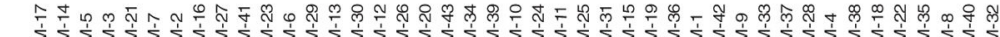

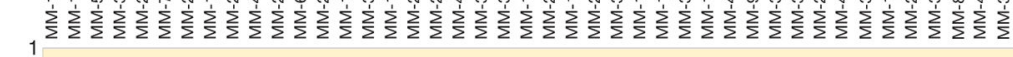

GII

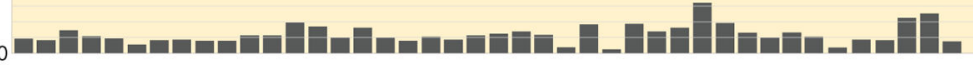

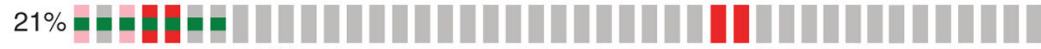

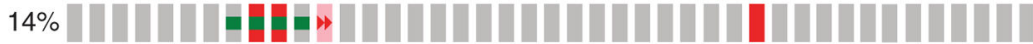

$7 \%$

$14 \%$

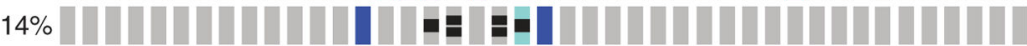

$26 \%$

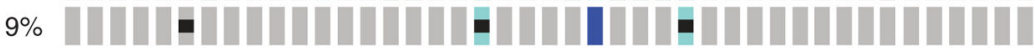

$9 \%$ E

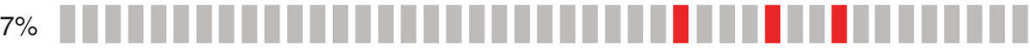

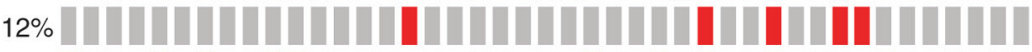

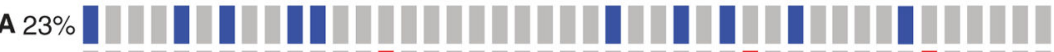

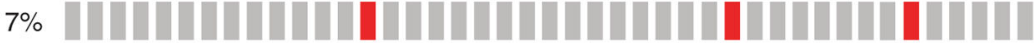

$12 \%$

7\%

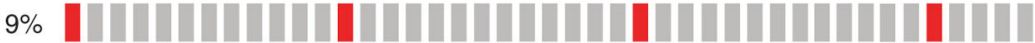
*

$16 \%$

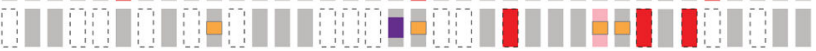

\footnotetext{
- Activating mutation - Truncating mutation $\mathbf{H}$ Homozygous truncating mutation $\square$ Promoter mutation

I Amplification Gain of mutant allele Deep Deletion Loss of wildtype allele

W Inframe Insertion Fusion in promoter $\square$ Gene not sequenced Promoter not sequenced
}
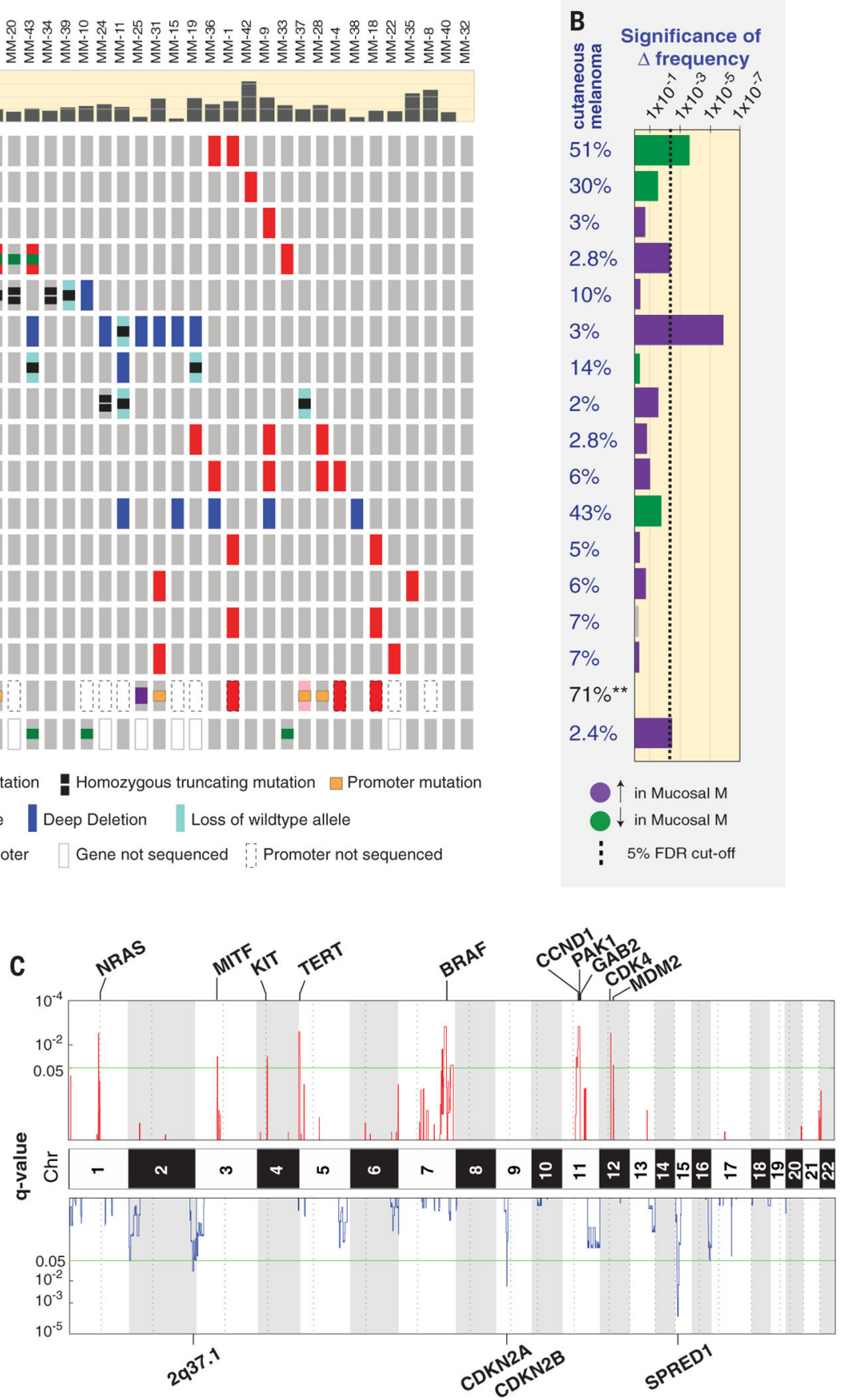

Fig. 1. Genetic alterations in 43 mucosal melanomas.

(A) Tiling plot summarizing pathogenic mutations, copy number alterations (CNAs), and structural variants in various genes (listed on left). Mucosal melanoma (MM) samples are arranged in columns. Gene alteration frequencies (including point mutations and CNAs) are shown as percentages. The bar graph on top shows the genome instability index (GII), which is the fraction of the genome affected by CNAs, for each sample. HIPPO, Hippo signaling pathway. (B) Mutation frequencies (which include pathogenic amplifications and deep deletions) from the TCGA skin cutaneous melanoma (SKCM) cohort are shown for 
comparison. The bar graph shows $\mathrm{P}$ values from Fisher's exact tests for significance of increased (purple) or decreased (green) mutation frequencies compared with the TCGA SKCM cohort. The dashed line marks the 5\% false detection rate (FDR) threshold. *TERT: $20 \%(4 / 20)$ activating promoter mutation, $7 \%$ (3/43) gene amplification, and 5\% (1/20) promoter structural rearrangement. **TERTpromoter mutation frequency in cutaneous melanomas (29). Mucosal M, mucosal melanoma. (C) GISTIC2 analysis identifies significant recurrent amplifications (red, top) and deletions (blue, bottom). Green lines indicate the q value thresholds for significance. $\mathrm{Chr}$, chromosome. 
A Chr 15

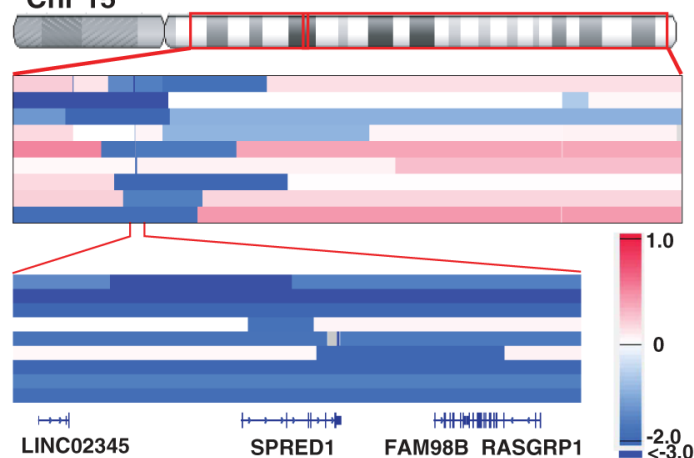

C

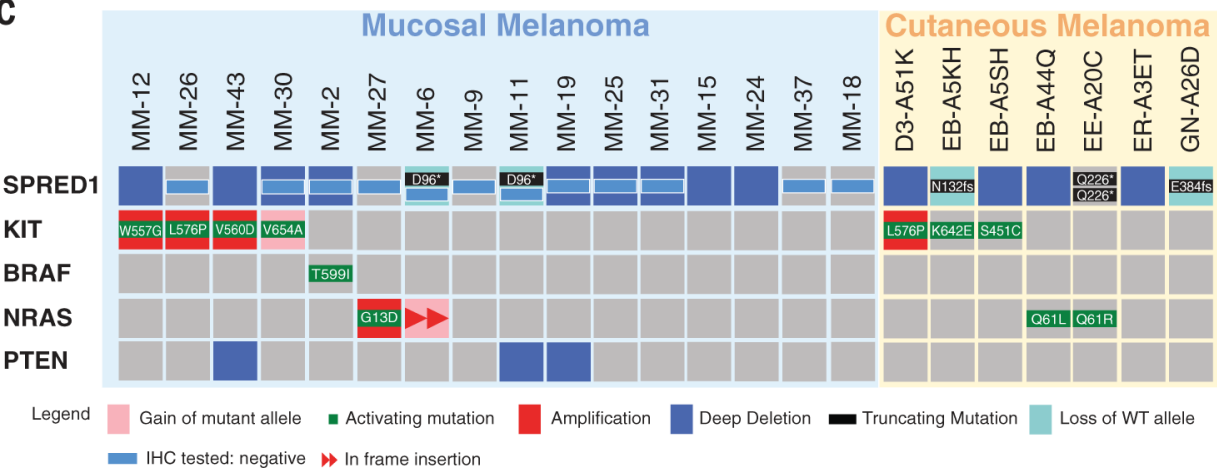

Fig. 2. Focused deletions at chromosome band $15 q 14$ nominate SPRED1 as a frequently lost tumor suppressor gene.

(A) Heat maps show SPRED1 deep deletion in nine tumors. $\log _{2}$-transformed copy number ratios are represented on a color scale. (B) SPRED1 immunohistochemistry (IHC) results for 26 tumors with available material. All samples with biallelic inactivation of SPRED1 loss were negative for SPRED1. *In one sample, weak SPRED1 staining was present in $25 \%$ of tumor cells, suggesting the presence of a subclonal tumor population that retains SPRED1. (C) Shown are mucosal melanoma and TCGA skin cutaneous melanoma cases that harbor SPRED1 biallelic loss-of-function mutations and/or exhibit loss of SPRED1 protein by IHC (for mucosal melanomas only), along with other MAPK pathway-activating mutations. In all cases where point mutations occur with gain or amplifications, the amplification occurs on the mutated allele. An asterisk indicates nonsense mutation. fs, frameshift; WT, wild type. Single-letter abbreviations for the amino acid residues are as follows: A, Ala; C, Cys; D, Asp; E, Glu; G, Gly; I, Ile; K, Lys; L, Leu; N, Asn; P, Pro; Q, Gln; R, Arg; S, Ser; T, Thr; V, Val; and W, Trp. 

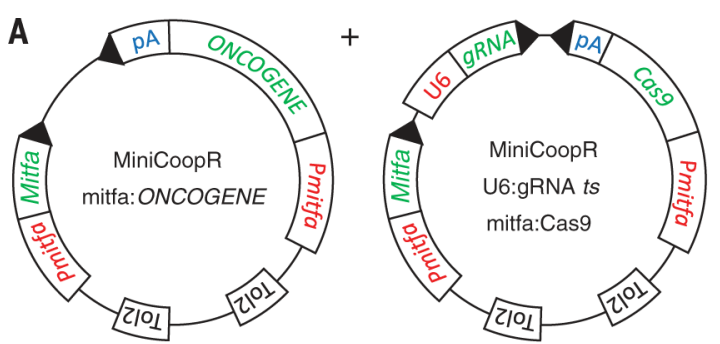

MiniCoopR mitfa: ONCOGENE MiniCoopR mitfa:Cas9, U6:gRNA ts Tol2 mRNA

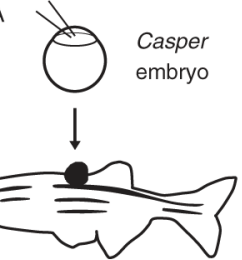

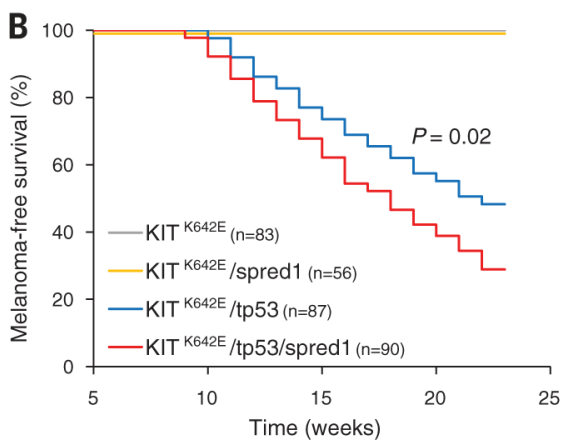
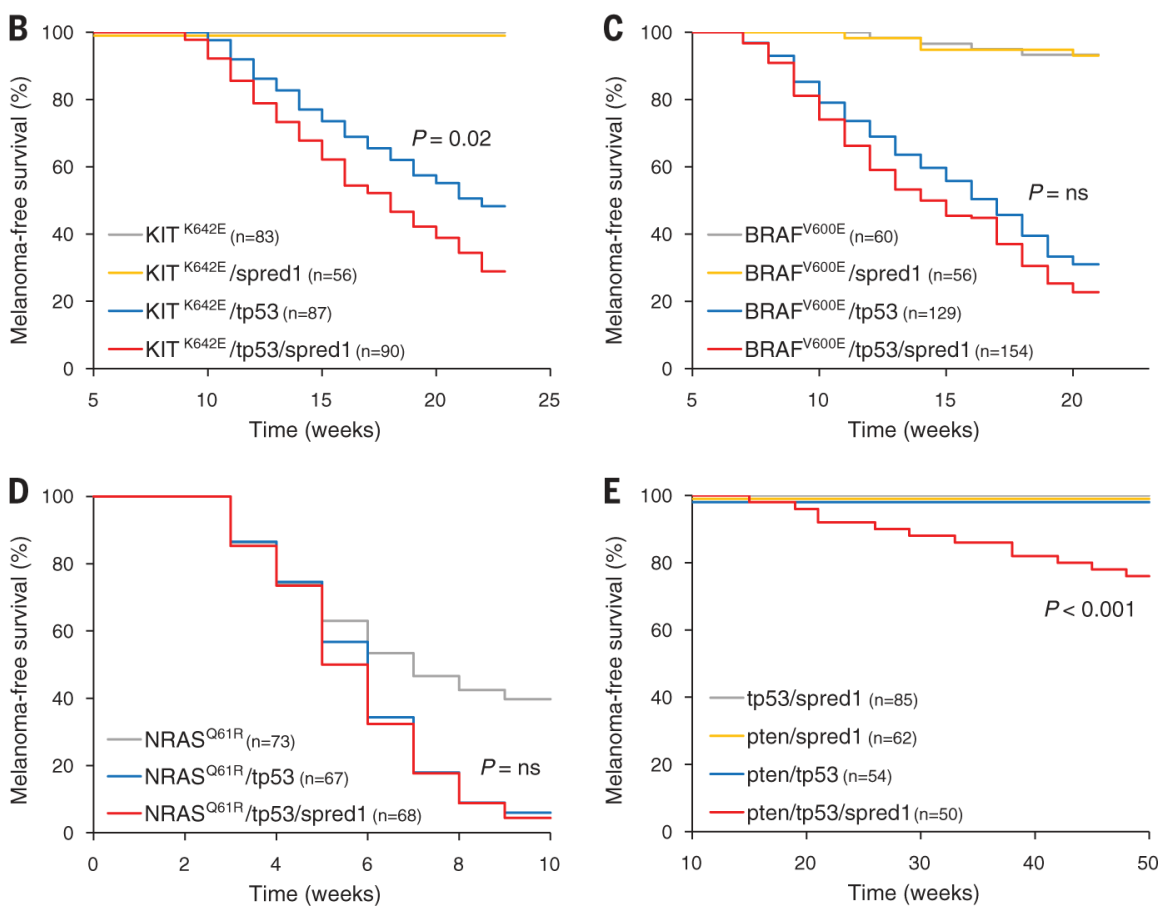

Fig. 3. Melanoma modeling in zebrafish establishes genetic cooperation between SPRED1 loss and KIT mutations.

(A) Schematic representation of the MiniCoopR vector (left) and the CRISPR MiniCoopR vector (right), enabling melanocyte-specific gene expression or inactivation, respectively. Pmitfa, mitfa promoter; pA, polyadenylation signal; ts, tumor suppressor. Injection of MiniCoopR or CRISPR MiniCoopR vectors into casper $\left(\mathrm{mitfa}^{-/-} ; \mathrm{roy}^{-/-}\right.$) embryos rescues melanocyte formation in a mosaic fashion in adult zebrafish. Combinations of vectors expressing the oncogenes $\mathrm{KIT}^{\mathrm{K} 642 \mathrm{E}}, \mathrm{BRAF}^{\mathrm{V} 600 \mathrm{E}}$, or NRAS ${ }^{\mathrm{Q} 1 \mathrm{R}}$ and targeting tp53 initiate melanoma. (B to $\mathbf{E}$ ) Melanoma-free survival curves of casper zebrafish injected with the indicated combinations of vectors expressing $\mathrm{KIT}^{\mathrm{K} 642 \mathrm{E}}, \mathrm{BRAF}^{\mathrm{V} 600 \mathrm{E}}$, or NRAS ${ }^{\mathrm{Q} 61 \mathrm{R}}$ and targeting tp53, pten, and/or spred1. P values were calculated by log-rank test. ns, not significant. 

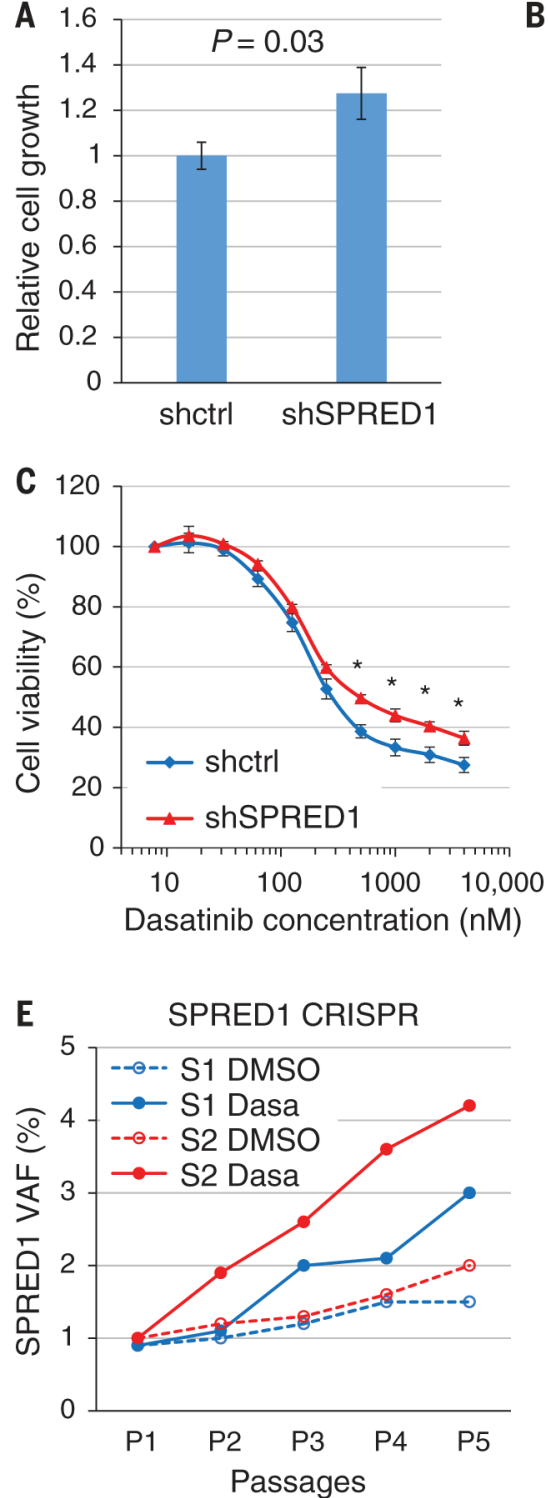
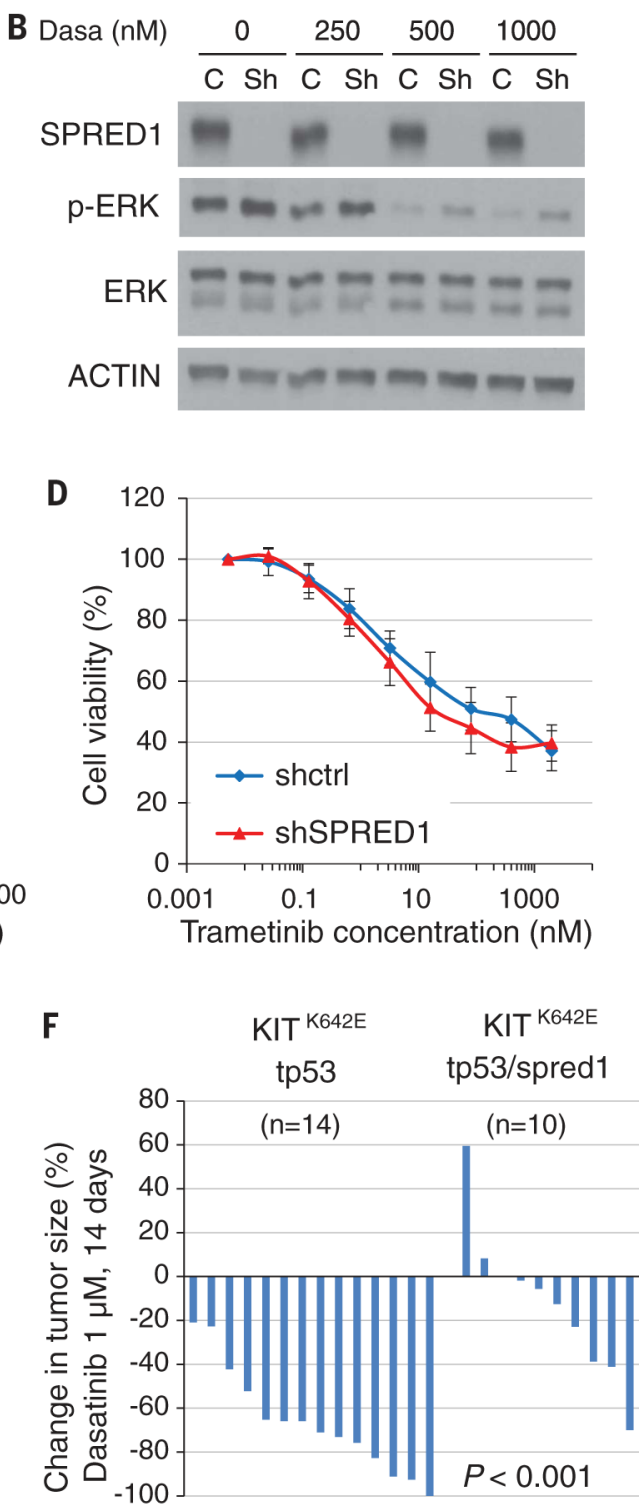

Fig. 4. Loss of SPRED1 confers resistance to KIT inhibition in KIT-mutant melanoma by sustaining MAPK signaling and cell proliferation.

(A) Proliferation of the KIT ${ }^{\mathrm{L} 576 \mathrm{P}}$-driven human melanoma cell line WM3211 stably expressing a control shRNA (shctrl) or a shRNA directed against SPRED1 (shSPRED1) measured after 4 days in culture and normalized to the control shRNA. Data are means \pm SD of three independent experiments, and $\mathrm{P}=0.03$ by paired Student's $\mathrm{t}$ test. (B) Western blot analysis of MAPK pathway activity in the cells described in (A) treated with the indicated concentrations of dasatinib (Dasa) for 6 hours. C, shctrl; Sh, shSPRED1; p-ERK, phosphorylated ERK. Actin was used as a loading control. (C and D) Viability of the cells described in (A) treated with increasing concentrations of the KIT inhibitor dasatinib (C) or the MEK inhibitor trametinib (D) for 4 days. Data are means \pm SD of three independent experiments, and $* \mathrm{P}<0.05$ by paired Student's $t$ test. (E) Evolution of frameshift variant allele fraction (VAF) in cultured WM3211 human melanoma cells transiently transfected with two independent vectors (S1 and S2) targeting SPRED1 by CRISPR and treated with 
either dimethyl sulfoxide (DMSO) or $500 \mathrm{nM}$ dasatinib over five passages. Data are from one representative experiment. (F) Quantification of tumor size in zebrafish injected with combinations of vectors expressing $\mathrm{KIT}^{\mathrm{K} 642 \mathrm{E}}$ and targeting either tp53 or both tp53 and spred1, after 14 days of $1 \mathrm{mM}$ dasatinib treatment, relative to tumor size before treatment. $\mathrm{P}<0.001$ by two-tailed Student's t test. 